\title{
Scalar conservation laws with rough (stochastic) fluxes: the spatially dependent case
}

\author{
Pierre-Louis Lions • Benoît Perthame • \\ Panagiotis E. Souganidis
}

Received: 17 March 2014 / Accepted: 26 July 2014 / Published online: 8 October 2014

(C) Springer Science+Business Media New York 2014

\begin{abstract}
We continue the development of the theory of pathwise stochastic entropy solutions for scalar conservation laws in $\mathbb{R}^{N}$ with quasilinear multiplicative "rough path" dependence by considering inhomogeneous fluxes and a single rough path like, for example, a Brownian motion. Following our previous note where we considered spatially independent fluxes, we introduce the notion of pathwise stochastic entropy solutions and prove that it is well posed, that is we establish existence, uniqueness and continuous dependence in the form of a (pathwise) $L^{1}$-contraction. Our approach is motivated by the theory of stochastic viscosity solutions, which was introduced and developed by two of the authors, to study fully nonlinear first- and second-order stochastic pde with multiplicative noise. This theory relies on special test functions constructed by inverting locally the flow of the stochastic characteristics. For conservation laws this is best implemented at the level of the kinetic formulation which we follow here.
\end{abstract}

\footnotetext{
P.-L. Lions

Collège de France and CEREMADE, Université de Paris-Dauphine, 1, Place Marcellin Berthelot, 75005 Paris Cedex 5, France

e-mail: lions@ceremade.dauphine.fr

B. Perthame $(\bowtie)$

Sorbonne Universités, UPMC, Univ. Paris 06, CNRS UMR 7598 Laboratoire J.-L. Lions,

BC 187, 4, Place Jussieu, 75252 Paris 5, France

e-mail: benoit.perthame@upmc.fr

B. Perthame

INRIA Paris-Rocquencourt, EPC Mamba, Le Chesnay, France

P. E. Souganidis

Department of Mathematics, University of Chicago, Chicago, IL 60637, USA

e-mail: souganidis@math.uchicago.edu
} 
Keywords Stochastic differential equations - Stochastic conservation laws . Stochastic entropy condition · Kinetic formulation · Dissipative solutions ·

Rough paths

Mathematics Subject Classification $\quad 35 \mathrm{~L} 65 \cdot 35 \mathrm{R} 60 \cdot 60 \mathrm{H} 15 \cdot 81 \mathrm{~S} 30$

\section{Introduction}

We continue the development of the theory of pathwise stochastic entropy solutions for scalar conservation laws in $\mathbb{R}^{N}$ with quasilinear multiplicative "rough path" dependence by considering inhomogeneous, that is spatially dependent, fluxes and a single rough path. In our previous note [17] we studied spatially independent fluxes and multiple paths.

Our approach is based on the concepts and methods introduced in [17] as well as by Lions and Souganidis in [20,21] and extended by the same authors in [22-25] for the theory of pathwise stochastic viscosity solution of fully nonlinear first- and secondorder stochastic pde including stochastic Hamilton-Jacobi equations. One of the fundamental tools of this theory is the class of test functions constructed by inverting locally, and at the level of test functions, the flow of the characteristics corresponding to the stochastic first-order part of the equation and smooth initial data. Such approach is best implemented for conservation laws using the kinetic formulation which we follow here.

It is important to remark that throughout the paper we use the term "rough path" for a non differentiable time dependent function and we do not make any connection with the Lyons [26] theory of rough paths since we are only considering a single path. We also note that to keep statements shorter, we often write sscl for scalar stochastic conservation laws any time we refer to equations of the type of study here.

Let

$$
\mathbf{A}=\left(A_{1}, \ldots, A_{N}\right) \in C^{2}\left(\mathbb{R}^{N} \times \mathbb{R} ; \mathbb{R}^{N}\right)
$$

be the flux and consider a single continuous "rough path", which may be, for example, a Brownian motion,

$$
W \in C([0, \infty) ; \mathbb{R}) \text { with } W(0)=0
$$

We are interested in the sscl

$$
\left\{\begin{array}{l}
d u+\operatorname{div} \mathbf{A}(x, u) \circ d W=0 \text { in } \mathbb{R}^{N} \times(0, \infty), \\
u=u^{0} \text { on } \mathbb{R}^{N} \times\{0\} .
\end{array}\right.
$$

Note that $u(x, t)=v(x, W(t))$, where $v$ solves a time-homogeneous equation $v_{t}+$ $\operatorname{div} \mathbf{A}(x, v)=0$, is formally a solution but it can not be an entropy solution because the change of time is incompatible with the formation of shocks.

Throughout the paper we adopt the notation and terminology of stochastic calculus. In general $d u$ denotes some kind of time differential, while, in the case that $W$ is Brownian, it is the usual stochastic differential. Similarly in the general setting o does not have any particular meaning and can be ignored, while in the stochastic setting it 
denotes the Stratonovich differential. The need to use the latter stems from the fact that we are developing a theory which is closed (stable) on paths in the local uniform topology and, in this context, Stratanovich is relevant. That a pathwise theory is more appropriate to study (3) is also justified from the fact that in the stochastic case, taking expectations leads, in view of the properties of the Ito calculus, to terms that are not possible to handle by the available estimates. We refer to [17] for an extended discussion of this point.

Our interest in sscl is twofold. Given the theory of stochastic viscosity solutions and the connection between conservation laws and Hamilton-Jacobi equations when $N=1$, it is very natural from the mathematical point of view to ask whether there is such a theory for the former. The second reason is that sccl like (3) arise naturally as models in the Lasry-Lions theory of mean field games [12-14]. We also refer to [17] for an example of stochastic system of interacting particles.

If, instead of (2), we assume that $W \in C^{1}\left((0, \infty) ; \mathbb{R}^{N}\right)$, then (3) is a "classical" problem with a well known theory; see, for example, the books of Dafermos [3] and Serre [30]. The solution can develop singularities in the form of shocks (discontinuities). Hence it is necessary to consider entropy solutions which, although not regular, satisfy the $L^{1}$-contraction property established by Kruzkov that yields uniqueness. Entropy solutions, which are based on certain inequalities, cannot be used when $\mathrm{W}$ is not smooth.

There are several challenges when trying to extend the analysis of [17] to spatially dependent fluxes. The underlying idea is the same, namely to use the characteristics at the kinetic level to "eliminate the bad stochastic terms". In the spatially independent setting the characteristics can be solved explicitly and, as a result, it is possible to keep track of all the cancellations that are taking place and, hence, obtain rather strong estimates. In the inhomogeneous setting there are no explicit solution of the system of the stochastic characteristics. As a result the calculations are not transparent and it becomes necessary to employ more complicated arguments to study the problem.

We remark that recently Debussche and Vovelle [7] (see also Feng and Nualart [9], Chen, Ding and Karlsen [2], Debussche, Hofmanová and Vovelle [6], Hofmanová [10,11], Berthelin and Vovelle [1] and Debussche and Vovelle [5]) put forward a theory of weak entropy solutions of scalar conservation laws with Ito-type semilinear stochastic dependence. Such problems do not appear to be amenable to a pathwise theory. Our results do not cover the equations studied in [7,9] and vice versa. We refer to Sect. 6 of [17] for a discussion of these issues.

\subsection{Organization of the paper}

The paper is organized as follows: In Sect. 2 we state the assumptions, we review briefly some facts about entropy solutions and the kinetic formulation and we introduce the notion of the pathwise solutions. In Sect. 3 we summarize some of the tools we use and prove a preliminary result. Sections 4 and 5 are devoted respectively to the proofs of the contraction and intrinsic uniqueness of the stochastic entropy solutions and their existence. In Sect. 5 we also state and prove general $L^{\infty}$ - and $L^{2}$-bounds. 


\section{The kinetic formulation and pathwise stochastic entropy solutions}

We consider the sscl (3). We write

$$
\operatorname{div} \mathbf{A}(x, u)=\operatorname{div}_{x} \mathbf{A}(x, u)+\operatorname{div}_{u} \mathbf{A}(x, u),
$$

where

$$
\operatorname{div}_{x} \mathbf{A}(x, u)=\sum_{i=1}^{N} A_{i, x_{i}}(x, u) \text { and } \operatorname{div}_{u} \mathbf{A}(x, u)=\sum_{i=1}^{N} A_{i, u}(x, u) u_{x_{i}}
$$

and introduce the notation

$$
\mathbf{a}(x, \xi)=\left(a_{1}(x, \xi), \ldots, a_{N}(x, \xi)\right):=\mathbf{A}_{u}(x, \xi)=\left(A_{1, u}(x, \xi), \ldots, A_{N, u}(x, \xi)\right)
$$

and

$$
b(x, \xi)=\operatorname{div}_{x} \mathbf{A}(x, \xi) .
$$

\subsection{Assumptions}

We summarize here the main assumptions we need in the paper. In addition to (1) and (2), we assume that

$$
b(x, 0)=0,
$$

and

$$
\partial_{j} a_{i}, \partial_{u} a_{i}, \partial_{j} b, \partial_{u} b \in L^{\infty}\left(\mathbb{R}^{N} \times \mathbb{R}\right) \quad \text { for } 1 \leq i, j \leq N
$$

\subsection{Entropy solutions for smooth paths and some estimates}

If $W \in C^{1}([0, \infty) ; \mathbb{R})$ and $u, v$ solve (3), the classical entropy inequalities yield

$$
d|u-v|+\operatorname{div}[[\mathbf{A}(x, u)-\mathbf{A}(x, v)] \operatorname{sgn}(u-v)] \circ d W \leq 0,
$$

and thus

$$
\frac{d}{d t} \int_{\mathbb{R}^{N}}|(u-v)(x, t)| d x \leq 0 .
$$

Since, in view of (6), $v \equiv 0$ is a solution, the contraction yields the a priori $L^{1}$-estimate

$$
\|u(\cdot, t)\|_{1} \leq\left\|u^{0}\right\|_{1}
$$

On the other hand $L^{\infty}$-estimates, which are also known as invariant regions, of the form

$$
\|u(\cdot, t)\|_{\infty} \leq C(t)
$$


for space dependent fluxes are not as general and require some additional work and stronger assumptions. We give a general argument in Sect. 5 and discuss some examples that are not covered by it.

\subsection{The kinetic formulation for smooth paths}

We review here the basic concepts of the kinetic theory of scalar conservation laws and we show that it allows to define a change of variable along the "kinetic" characteristics which turns out to be a very convenient tool for the study of sscl.

Although we use the notation of the introduction, here we assume that $W \in$ $C^{1}([0, \infty) ; \mathbb{R})$, in which case $d u$ stands for the usual derivative and $\circ$ is the usual multiplication and, hence, should be ignored.

The entropy inequalities (see, for example, $[3,30]$ ), which yield (8) and guarantee the uniqueness of the entropy solutions, are

$$
\left\{\begin{array}{l}
d S(u)+\left[\operatorname{div}_{u}\left(\mathbf{A}^{S}(x, u)\right)+S^{\prime}(u)\left[\operatorname{div}_{x} \mathbf{A}\right](x, u)-B^{S}(x, u)\right] \circ d W \\
\quad \leq 0 \text { in } \mathbb{R}^{N} \times(0, \infty) \\
S(u)=S\left(u^{0}\right) \quad \text { on } \mathbb{R}^{N} \times\{0\}
\end{array}\right.
$$

for all $C^{2}$-convex functions $S$, entropy fluxes $\mathbf{A}^{\mathbf{S}}$ and forcing terms $B^{S}$ defined by

$$
\mathbf{A}^{\mathbf{S}}(x, u)_{u}=\mathbf{A}_{u}(x, u) S^{\prime}(u) \quad \text { and } \quad B^{S}(x, u)_{u}=\operatorname{div} \mathbf{A}_{u}(x, u) S^{\prime}(u)
$$

It is by now well established that the simplest way to study conservation laws is to use the kinetic formulation developed in a series of papers - see Perthame and Tadmor [29], Lions, Perthame and Tadmor [18], Perthame [27,28], and Lions, Perthame and Souganidis $[15,16]$. The kinetic formulation for inhomogeneous in space but time independent conservation laws was developed by Dalibard [4]. The arguments of [4] easily extend to problems with smooth multiplicative paths.

The idea is to write a linear equation for the nonlinear function $\chi: \mathbb{R}^{N} \times \mathbb{R} \times$ $(0, \infty) \rightarrow \mathbb{R}$ given by

$$
\chi(x, \xi, t):=\chi(u(x, t), \xi)=\left\{\begin{array}{cc}
+1 & \text { if } 0 \leq \xi \leq u(x, t) \\
-1 & \text { if } u(x, t) \leq \xi \leq 0 \\
0 & \text { otherwise }
\end{array}\right.
$$

The kinetic formulation says that, if $u^{0} \in\left(L^{1} \cap L^{\infty}\right)\left(\mathbb{R}^{N}\right)$, the set of entropy inequalities (11) for all convex entropies $S$ can be replaced by the equation

$$
\left\{\begin{array}{l}
d \chi+\operatorname{div}_{x}[\mathbf{a}(x, \xi) \chi] \circ d W-\frac{\partial}{\partial \xi}[b(x, \xi) \chi] \circ d W=\partial_{\xi} m d t \text { in } \mathbb{R}^{N} \times \mathbb{R} \times(0, \infty), \\
\chi=\chi\left(u^{0}(\cdot), \cdot\right) \text { on } \mathbb{R}^{N} \times \mathbb{R}^{2} \times\{0\},
\end{array}\right.
$$


which is supposed to be satisfied in the sense of distributions, where

$$
m \text { is a nonnegative measure in } \mathbb{R}^{N} \times \mathbb{R} \times(0, \infty)
$$

and

$$
\left\{\begin{array}{l}
\int_{0}^{T} \int_{\mathbb{R}} \int_{\mathbb{R}^{N}} m(x, \xi, t) d x d \xi d t \leq \frac{1}{2}\left\|u^{0}\right\|_{2}+\int_{0}^{T} \int_{\mathbb{R}} \int_{\mathbb{R}^{N}}|b(x, \xi) \| \dot{W}| \\
\quad \chi d \xi d x d t \text { for all } T>0 \text { and } \\
m(x, \xi, t)=0 \text { for }|\xi| \geq\|u(\cdot, t)\|_{\infty}
\end{array}\right.
$$

note that the estimate on mass is new even in the context of the "classical" conservation laws, that is without rough path dependence.

We remark that although to prove the equivalence between entropy and kinetic solutions it is necessary to work in $\left(L^{1} \cap L^{\infty}\right)\left(\mathbb{R}^{N}\right)$, the kinetic theory of [4] is set only in $L^{1}$. Hence the condition on the support of the measure, which is equivalent to an $L^{\infty}$-bound for $u$, is not needed provided it is assumed that $b \in L^{1}\left(\mathbb{R}^{N} \times \mathbb{R}\right)$. More importantly we note that a priori bound for the total mass of the measure in (15) is useless for irregular paths since it depends on the total variation of the path.

Using some of the ideas in this paper it is, however, possible to improve the upper bound so that it becomes independent of the variation of the path. In Sect. 5 we sketch the proof of such an estimate as well as an $L^{\infty}$-bound on the solutions. We summarize these in

$$
\left\{\begin{array}{l}
\text { there exists } C_{T}>0 \text { such that } \int_{0}^{T} \int_{\mathbb{R}} \int_{\mathbb{R}^{N}} m(x, \xi, t) d x d \xi d t \leq C_{T}\left\|u^{0}\right\|_{2}^{2}, \\
\text { and } \\
m(x, \xi, t)=0 \text { for }|\xi| \geq\|u(\cdot, t)\|_{\infty} .
\end{array}\right.
$$

Notice that the transport equation for $\chi$ has a Hamiltonian type structure and is equivalently written in the strong form

$$
d \chi+\mathbf{a}(x, \xi) \cdot D_{x} \chi \circ d W-b(x, \xi) D_{\xi} \chi \circ d W=\partial_{\xi} m d t
$$

For $\rho^{0} \in C_{\mathrm{b}}^{\infty}\left(\mathbb{R}^{N} \times \mathbb{R}\right)$ and $t_{0} \geq 0$, let $\rho\left(\cdot, \cdot, \cdot ; t_{0}\right)$ be the solution of the linear stochastic pde

$$
\left\{\begin{array}{l}
d \rho+\left[\mathbf{a}(x, \xi) \cdot D_{x} \rho-b(x, \xi) D_{\xi} \rho\right] \circ d W=0 \text { in } \mathbb{R}^{N} \times \mathbb{R} \times\left(t_{0}, \infty\right), \\
\rho=\rho^{0} \text { on } \mathbb{R}^{N} \times \mathbb{R} \times\left\{t_{0}\right\}
\end{array}\right.
$$

Since $W$ is a single path, it is immediate that

$$
\rho\left(x, \xi, t ; t_{0}\right)=\hat{\rho}\left(x, \xi, W(t)-W\left(t_{0}\right)\right)
$$

where, in view of the assumptions above, $\hat{\rho}$ is the smooth solution of 


$$
\left\{\begin{array}{l}
\hat{\rho}_{t}+\mathbf{a}(x, \xi) \cdot D_{x} \hat{\rho}-b(x, \xi) D_{\xi} \hat{\rho}=0 \text { in } \mathbb{R}^{N} \times \mathbb{R} \times \mathbb{R} \\
\hat{\rho}=\rho^{0} \text { on } \mathbb{R}^{N} \times \mathbb{R} \times\{0\}
\end{array}\right.
$$

Next for $\rho^{0} \in C_{\mathrm{b}}^{\infty}\left(\mathbb{R}^{N} \times \mathbb{R}\right), t_{0} \geq 0$ and $y, \eta \in \mathbb{R}^{N}$ we consider the solution $\rho\left(\cdot, \cdot, y, \eta, \cdot ; t_{0}\right)$ of $(18)$ with initial datum, at $t=t_{0}, \rho^{0}(\cdot-y, \cdot-\eta)$ and introduce the "convolution" along characteristics given by

$$
\rho \star \chi\left(y, \eta, t ; t_{0}\right):=\int \rho\left(x, \xi, y, \eta, t ; t_{0}\right) \chi(x, \xi, t) d x d \xi
$$

Combining (17) and (18) we find that, in the sense of distributions,

$$
\frac{d}{d t} \rho \star \chi\left(y, \eta, t ; t_{0}\right)=-\int \partial_{\xi} \rho\left(x, \xi, y, \eta, t ; t_{0}\right) m(x, \xi, t) d x d \xi
$$

It follows that, for all almost every $t_{0} \geq 0$ and almost all $t \geq 0$,

$$
\rho \star \chi(y, \eta, t)=\rho \star \chi^{0}\left(y, \eta, t_{0} ; t_{0}\right)-\int_{t_{0}}^{t} \int \partial_{\xi} \rho(x, \xi, y, \eta, t) m(x, \xi, s) d x d \xi d s
$$

The $L^{1}$-continuity of $u$ at $t=0$, as stated later, implies that this formula holds for $t_{0}=0$ and for all almost every $t \geq 0$.

Note that although the regularity of the path was used to derive (21) and (22), the actual statements do not need it and make sense for paths which are only continuous. Notice also that (21) and (22) are equivalent to the kinetic formulation when the measure $m$ satisfies (14) and (16).

\subsection{Pathwise stochastic entropy solutions}

Neither the notion of entropy solution nor the kinetic formulation can be used to study (3), since both involve either entropy inequalities or the sign of the defect measure quantities which do not make sense for equations/expressions with, in principle, are nowhere differentiable functions. We refer to $[20,21,23,24]$ for a general discussion about the difficulties encountered when attempting to use the classical weak solution approaches to study fully nonlinear stochastic pde.

Motivated by our previous work [17] as well as the theory of stochastic viscosity solutions $[20,21,23,24]$ we use (21) to introduce next the notion of pathwise stochastic entropy solutions for SSCL. Recall, as remarked previously, that the statement of (21) does not rely on any regularity of the paths. We have:

Definition 2.1 Assume (1), (2), (6) and (7). Then $u \in\left(L^{1} \cap L^{\infty}\right)\left(\mathbb{R}^{N} \times(0, T)\right)$, for all $T>0$, is a pathwise stochastic entropy solution of (3), if there exists a nonnegative bounded measure $m$ on $\mathbb{R}^{N} \times \mathbb{R} \times(0, \infty)$ satisfying (16) such that (21) and (22) hold for all $\rho$ 's given by (20) with $\rho^{0} \in C_{\mathrm{b}}^{\infty}\left(\mathbb{R}^{N} \times \mathbb{R}\right)$, almost everywhere in $\left(t_{0}, T\right)$, for almost all $t_{0} \geq 0$ and for $t_{0}=0$. 
We show in the next two sections that the pathwise stochastic entropy solutions exist and satisfy a contraction in $L^{1}$ and, hence, are stable and unique.

\section{Some technical results}

We discuss here the class of test functions we use in the paper, recall the method of characteristics which provide a representation for the solutions to (18) and (19) and show what they imply for the special solutions we are considering, and, finally, state and prove a technical result.

\subsection{The test functions}

For each $\varepsilon>0$ we consider test functions solving solving (17) with initial data $\rho_{\varepsilon}^{0}$ that separates space, $x \in R^{N}$, and velocity, $\xi \in \mathbb{R}$. A natural choice is convolution approximation to Dirac masses, that is

$$
\rho_{\varepsilon}^{0}(x-y, \xi-\eta)=\rho_{\varepsilon}^{s}(x-y) \rho_{\varepsilon}^{v}(\xi-\eta),
$$

where, as $\varepsilon \rightarrow 0$ and in the sense of distributions,

$$
\rho_{\varepsilon}^{s} \longrightarrow \delta(x-y) \text { and } \rho_{\varepsilon}^{v} \longrightarrow \delta(\xi-\eta) ;
$$

here we use the superscripts $s$ and $v$ to signify whether the initial data approximates the space or the velocity variables. Moreover $\delta_{a}$ is the Dirac mass at $a$ and we write $\delta$ if $a=0$. Typical choices for $\rho_{\varepsilon}^{s}$ and $\rho_{\varepsilon}^{v}$ are

$$
\rho_{\varepsilon}^{s}(x)=\varepsilon^{-N} \rho^{s}(x / \varepsilon) \text { and } \rho_{\varepsilon}^{v}(\xi)=\varepsilon^{-1} \rho^{v}(\xi / \varepsilon),
$$

for some smooth functions $\rho^{s}$ and $\rho^{v}$ with compact support of diameter 1 and such that

$$
0 \leq \rho^{s}, \rho^{v} \leq 1, \rho^{s}(0)=\rho^{v}(0)=1 \text { and } \int_{\mathbb{R}^{N}} \rho^{s}=\int_{\mathbb{R}} \rho^{v}=1
$$

\subsection{The characteristics}

Depending on the context we use the forward and backwards characteristics of (19) to construct the smoothing kernels $\rho_{\varepsilon}$ and $\hat{\rho}_{\varepsilon}$, that is the solutions to (18) and (19) respectively.

Using the forward characteristics

$$
\begin{cases}\dot{Y}_{(y, \eta)}(s)=\mathbf{a}\left(Y_{(y, \eta)}(s), \zeta_{(y, \eta)}(s)\right), & Y_{(y, \eta)}(0)=y \\ \dot{\zeta}_{(y, \eta)}(s)=-b\left(Y_{(y, \eta)}(s), \zeta_{(y, \eta)}(s)\right), & \zeta_{(y, \eta)}(0)=\eta\end{cases}
$$

we generate the smoothing kernels $\widehat{\rho}_{\varepsilon}$ and $\rho_{\varepsilon}$ and which satisfy, in the sense of distributions,

$$
\widehat{\rho}_{\varepsilon}(x, \xi, y, \eta, s) \underset{\varepsilon \rightarrow 0}{\longrightarrow} \delta\left(x-Y_{(y, \eta)}(s)\right) \delta\left(\xi-\zeta_{(y, \eta)}(s)\right)
$$


and

$$
\rho_{\varepsilon}(x, \xi, y, \eta, t) \underset{\varepsilon \rightarrow 0}{\longrightarrow} \delta\left(x-Y_{(y, \eta)}\left(W(t)-W\left(t_{0}\right)\right)\right) \delta\left(\xi-\zeta_{(y, \eta)}\left(W(t)-W\left(t_{0}\right)\right)\right)
$$

We may also use the backward characteristics, that is the solution of

$$
\begin{cases}\dot{X}_{(t, x, \xi)}(s)=\mathbf{a}\left(X_{(t, x, \xi)}, \Xi_{(t, x, \xi)}\right), & X_{(t, x, \xi)}(t)=x \\ \dot{\Xi}_{(t, x, \xi)}(s)=-b\left(X_{(t, x, \xi)}, \Xi_{(s, x, \xi)}\right), & \Xi_{(t, x, \xi)}(t)=\xi\end{cases}
$$

then

$$
\widehat{\rho}_{\varepsilon}(x, \xi, y, \eta, t)=\rho_{\varepsilon}^{0}\left(X_{(t, x, \xi)}(0)-y, \Xi_{(t, x, \xi)}(0)-\eta\right)
$$

and

$$
\rho_{\varepsilon}\left(x, \xi, y, \eta, t ; t_{0}\right)=\rho_{\varepsilon}^{0}\left(X_{\left(W(t)-W\left(t_{0}\right), x, \xi\right)}(0)-y, \Xi_{\left(W(t)-W\left(t_{0}\right), x, \xi\right)}(0)-\eta\right)
$$

We note that, in view of (6) and the uniqueness of the solutions to (25) and (28),

$$
\zeta_{(y, 0)}(t)=\Xi_{(t, x, 0)}=0, \operatorname{sgn}\left(\zeta_{(y, \eta)}(t)\right)=\operatorname{sgn}(\eta) \text { and } \operatorname{sgn}\left(\Xi_{(t, x, \xi)}(s)\right)=\operatorname{sgn}(\xi) \text {. }
$$

To keep the notation simple in the sequel we write sometimes $\left(X_{(x, \xi)}, \Xi_{(x, \xi)}\right)$ for $\left(X_{\left(W(t)-W\left(t_{0}\right), x, \xi\right)}(0), \Xi_{\left(W(t)-W\left(t_{0}\right), x, \xi\right)}(0)\right)$ and

$$
\rho_{\varepsilon}(x, \xi, y, \eta, t)=\rho_{\varepsilon}^{s}\left(X_{(x, \xi)}-y\right) \rho_{\varepsilon}^{v}\left(\Xi_{(x, \xi)}-\eta\right) .
$$

\subsection{Some technical facts}

We present now two technical facts which we will use in the next section. It concerns the behavior of

$$
\bar{q}_{\varepsilon}\left(x, \xi, t ; t_{0}\right):=\int_{\mathbb{R}^{N}} \int_{\mathbb{R}} q_{\varepsilon}\left(x, \xi, y, \eta, t ; t_{0}\right) d y d \eta \text { and } \partial_{\xi} \bar{q}_{\varepsilon}\left(x, \xi, t ; t_{0}\right),
$$

where, for each $y \in \mathbb{R}^{N}$ and $\eta \in \mathbb{R}, q_{\varepsilon}\left(x, \xi, y, \eta, t ; t_{0}\right)$ is the solution of (18) starting with initial datum

$$
q_{\varepsilon}\left(x, \xi, y, \eta, t_{0} ; t_{0}\right):=\rho_{\varepsilon}^{s}(x-y) \rho_{\varepsilon}^{v}(-\eta)\left(-\frac{1}{2}+\int_{-\infty}^{\xi} \rho_{\varepsilon}^{v}(\bar{\xi}-\eta) d \bar{\xi}\right)
$$

it is, of course, immediate that $\bar{q}_{\varepsilon}\left(\cdot, \cdot, \cdot ; t_{0}\right)$ is the solution of (18) with initial datum

$$
\bar{q}_{\varepsilon}\left(x, \xi, t_{0} ; t_{0}\right)=-\frac{1}{2}+\int_{\mathbb{R}} \int_{-\infty}^{\xi} \rho_{\varepsilon}^{v}(\bar{\xi}-\eta) \rho_{\varepsilon}^{v}(-\eta) d \bar{\xi} d \eta .
$$

The result is: 
Lemma 3.1 Assume (1), (2), (6) and (7) and, for $t_{0} \geq 0$ and $\varepsilon>0$, let $\bar{q}_{\varepsilon}\left(\cdot, \cdot, \cdot ; t_{0}\right)$ be given by (30). As $\varepsilon \rightarrow 0$, for all times $t$ and a.e. in $(x, \xi), \bar{q}_{\varepsilon}\left(x, \xi, t ; t_{0}\right) \rightarrow \frac{1}{2} \operatorname{sign}(\xi)$ and, thus, in $L_{\text {loc }}^{p}\left(\mathbb{R}^{N} \times \mathbb{R}\right)$ for all $p \in[1, \infty)$.

Proof In view of the relationship between the solutions to (18) and (19), it suffices to prove the claim for the solution $\hat{q}_{\varepsilon}$ of (19) with $\hat{q}_{\varepsilon}(x, \xi, y, \eta, 0)$ as in (31).

In light of the discussion in the previous paragraph, for each fixed $t$, we have

$$
\begin{aligned}
\int_{\mathbb{R}^{N}} \int_{\mathbb{R}} \hat{q}_{\varepsilon}(x, \xi, y, \eta, t) d y d \eta= & \int_{\mathbb{R}^{N}} \int_{\mathbb{R}} \hat{q}_{\varepsilon}\left(X_{(t, x, \xi)}(0), \Xi_{(t, x, \xi)}(0), y, \eta, 0\right) d y d \eta \\
= & -\frac{1}{2}+\int_{\mathbb{R}} \int_{-\infty}^{\Xi_{(t, x, \xi)}(0)} \rho_{\varepsilon}^{v}(\bar{\xi}-\eta) \rho_{\varepsilon}^{v}(-\eta) d \bar{\xi} d \eta \\
& \underset{\varepsilon \rightarrow 0}{\longrightarrow}-\frac{1}{2}+\mathbf{1}_{\left\{\Xi_{(t, x, \xi)}(0)>0\right\}}=-\frac{1}{2}+\mathbf{1}_{\{\xi>0\}},
\end{aligned}
$$

the last equality being a consequence of (29).

As far as $\partial_{\xi} \bar{q}_{\varepsilon}\left(x, \xi, t ; t_{0}\right)$ is concerned we first observe that

$$
\begin{aligned}
\partial_{\xi} \hat{q}_{\varepsilon}\left(x, \xi, y, \eta, t ; t_{0}\right)= & -\rho_{\varepsilon}^{v}(-\eta) D_{y} \rho_{\varepsilon}^{s}\left(X_{(t, x, \xi)}(0)-y\right)\left[-\frac{1}{2}\right. \\
& \left.+\int_{-\infty}^{\Xi_{(t, x, \xi)}(0)} \rho_{\varepsilon}^{v}(\bar{\xi}-\eta) d \bar{\xi}\right] \partial_{\xi} X_{(t, x, \xi)}(0) \\
& +\rho_{\varepsilon}^{v}(-\eta) \rho_{\varepsilon}^{s}\left(X_{(t, x, \xi)}(0)-y\right) \rho_{\varepsilon}^{v}\left(\Xi_{(t, x, \xi)}(0)-\eta\right) \partial_{\xi} \Xi_{(t, x, \xi)}(0) .
\end{aligned}
$$

Integrating in $y$ and $\eta$ we find

$$
\partial \xi \bar{q}_{\varepsilon}\left(x, \xi, t ; t_{0}\right)=\int_{\mathbb{R}^{N}} \int_{\mathbb{R}} \rho_{\varepsilon}^{v}(-\eta) \rho_{\varepsilon}\left(x, \xi, y, \eta, t ; t_{0}\right) d \eta d y \partial_{\xi} \Xi_{\left(W(t)-W\left(t_{0}\right), x, \xi\right)}(0)
$$

\section{The stability and uniqueness of pathwise stochastic entropy solutions}

\subsection{The result}

The result about the $L^{1}$-contraction property and, hence, the intrinsic uniqueness of the pathwise stochastic entropy solutions, is stated next.

Theorem 4.1 Assume (1), (2), (6), (7) and $u^{0} \in\left(L^{1} \cap L^{\infty}\right)\left(\mathbb{R}^{N}\right)$ and fix $T>0$. There exists at most one pathwise stochastic entropy solution $u \in L^{\infty}\left((0, T) ; L^{1} \cap L^{\infty}\left(\mathbb{R}^{N}\right)\right)$ to (3) which is continuous at $t=0$ with values in $L^{1}\left(\mathbb{R}^{N}\right)$. In addition any two pathwise stochastic entropy solutions $u_{1}, u_{2}$ satisfy, for almost all $t>0$, the "contraction" property

$$
\left\|u_{2}(\cdot, t)-u_{1}(\cdot, t)\right\|_{1} \leq\left\|u_{2}^{0}-u_{1}^{0}\right\|_{1} .
$$




\subsection{The proof}

When $W$ is smooth, the proof of (33) is based on considering, for two solutions $u_{1}$ and $u_{2}$ and $\chi^{(i)}(x, \xi, t)=\chi\left(u_{i}(x, t) ; \xi\right)$, the function

$$
\begin{aligned}
F(t) & :=\int\left[\left|\chi^{(1)}(x, \xi, t)\right|+\left|\chi^{(2)}(x, \xi, t)\right|-2 \chi^{(1)}(x, \xi, t) \chi^{(2)}(x, \xi, t)\right] d x d \xi \\
& =\left\|u_{1}(t)-u_{2}(t)\right\|_{1}
\end{aligned}
$$

and showing that $d F / d t \leq 0$, which yields the contraction property

$$
F\left(t_{2}\right) \leq F\left(t_{1}\right) \text { for almost all } 0 \leq t_{1} \leq t_{2}
$$

This is, however, not possible for the paths we are considering in this note. It is, therefore, necessary to modify the integrand in $F(t)$ in order to make use of the defining properties of stochastic entropy solutions. In particular we need to replace $\left|\chi\left(u_{i}(x, t), \xi\right)\right|=\operatorname{sign}(\xi) \chi\left(u_{i}(x, t), \xi\right)$ by the "convolution" along characteristics (20) for an appropriate choice of $\rho$, which is going to be the $q_{\varepsilon}\left(\cdot, \cdot, \cdot ; t_{0}\right)$ in (30). We also need to localize in time and use an iteration. The reason is that the approximation creates errors which can be controlled by the oscillations of the path $W$. Thus we need to discretize in time and, hence, to use $\rho_{\varepsilon}$ 's and $q_{\varepsilon}$ 's starting at different times and to add the errors.

We present now the

The proof of Theorem 4.1 The uniqueness follows immediately from the contraction property, hence, here we concentrate on the latter. Since the proof is long and technical we divide it in several steps some of which, although formally true, require justification.

The general set up. Fix $T>0$. We begin with a regularization of the functional $F$. For $t_{0} \geq 0, \varepsilon>0$ small, for $t \geq t_{0}$ and $q_{\varepsilon}$ as in Lemma 3.1, let

$$
\begin{aligned}
F_{t_{0}, \varepsilon}(t):= & \int\left[q_{\varepsilon}\left(\cdot, t ; t_{0}\right) \star \chi^{(1)}(y, \eta, t)+q_{\varepsilon}\left(\cdot, t ; t_{0}\right) \star \chi^{(2)}(y, \eta, t)\right. \\
& \left.-2 \rho_{\varepsilon}\left(\cdot, t ; t_{0}\right) \star \chi^{(1)}(y, \eta, t) \rho_{\varepsilon}\left(\cdot, t ; t_{0}\right) \star \chi^{(2)}(y, \eta, t)\right] d y d \eta .
\end{aligned}
$$

It follows from the a.e. continuity in time of the $F$ as well as (27) that, for almost every $t \in[0, T]$ as $\varepsilon \rightarrow 0$,

$$
F_{t_{0}, \varepsilon}(t) \rightarrow F(t)
$$

For $h>0$, let $\omega(h)$ denote the oscillation of the path $W$ over time intervals of size $h$, that is

$$
\omega(h):=\sup _{0 \leq s \leq h, 0 \leq t \leq T}|W(t+s)-W(t)| .
$$

Fix $s, t$ so that $0 \leq s \leq t \leq T$ and let $\Delta=\left\{s=t_{0} \leq t_{1} \leq \cdots \leq t_{M}=t\right\}$ be a partition of $[s, t]$ with mesh size $h=t_{i+1}-t_{i}$ and such that (34) holds for all $t_{i} \in \Delta$. 
The conclusion follows if we show that there exists $C>0$ such that

$$
F_{t_{i}, \varepsilon}\left(t_{i+1}\right)-F_{t_{i}, \varepsilon}\left(t_{i}\right) \leq C \omega(h) \int_{t_{i}}^{t_{i+1}} \int_{\mathbb{R}^{N}} \int_{\mathbb{R}}\left[m^{(1)}(x, \xi, t)+m^{(2)}(x, \xi, t)\right] d \xi d x d t .
$$

Indeed if (36) holds, then

$$
\begin{aligned}
F(t)-F(s) & =\sum_{i=0}^{M-1}\left[F\left(t_{i+1}-F\left(t_{i}\right)\right]=\lim _{\varepsilon \rightarrow 0} \sum_{i=0}^{M-1}\left[F_{t_{i}, \varepsilon}\left(t_{i+1}\right)-F_{t_{i}, \varepsilon}\left(t_{i}\right)\right]\right. \\
& \leq C \omega(h) \int_{s}^{t} \int_{\mathbb{R}^{N}} \int_{\mathbb{R}}\left[m^{(1)}(x, \xi, t)+m^{(2)}(x, \xi, t)\right] d \xi d x d t
\end{aligned}
$$

In view of (16), to prove (36) it suffices to show that, for almost every $t_{0} \geq 0$ and $h, \varepsilon>0$, there exists $C>0$ such that almost everywhere in $\left(t_{0}, t_{0}+h\right)$

$$
\frac{d F_{t_{0}, \varepsilon}}{d t} \leq C \omega(h) \int_{\mathbb{R}^{N}} \int_{\mathbb{R}} m(x, \xi, t) d \xi d x
$$

notice that this can be written only for continuity times of the right hand side, which include $t_{0}=0$ and this is enough for our purpose.

Since the upper bound for $d F_{t_{0}, \varepsilon} / d t$ requires some long and tedious calculations, we divide the computations and estimates in several parts. In the first, which is the longest, we estimate the derivative of the product term. The second is about about the single terms. After grouping everything together we find the error term. In what follows, to keep the notation simple, we assume that $t_{0}=0$, write $F_{\varepsilon}$ instead of $F_{0, \varepsilon}$, we omit the dependence of the solutions of (18) and (19) on the initial time and we write $X_{(x, \xi)}, \Xi_{(x, \xi)}, X_{\left(x^{\prime}, \xi^{\prime}\right)}$ and $\Xi_{\left(x^{\prime}, \xi^{\prime}\right)}$ in place of $X_{(W(t), x, \xi)}(0), \Xi_{(W(t), x, \xi)}(0), X_{\left(W(t), x^{\prime}, \xi^{\prime}\right)}(0)$ and $\Xi_{\left(W(t), x^{\prime}, \xi^{\prime}\right)}(0)$.

The product term in $\frac{d}{d t} F_{\varepsilon}$. It follows from the definitions that

$$
-\frac{d}{d t}\left[\rho_{\varepsilon} \star \chi^{(1)}(y, \eta, t) \rho_{\varepsilon} \star \chi^{(2)}(y, \eta, t)\right]=I_{1}(t)+I_{2}(t)
$$

where

$$
\left\{\begin{array}{l}
I_{1}(t):=\int \partial_{\xi} \rho_{\varepsilon}(x, \xi, y, \eta, t) m^{(1)}(x, \xi, t) \rho_{\varepsilon}\left(x^{\prime}, \xi^{\prime}, y, \eta, t\right) \chi^{(2)}\left(x^{\prime}, \xi^{\prime}, t\right) d x d \xi d x^{\prime} d \xi^{\prime}, \\
\text { and } \\
I_{2}(t):=\int \rho_{\varepsilon}(x, \xi, y, \eta, t) \chi^{(1)}(x, \xi, t) \partial_{\xi} \rho_{\varepsilon}\left(x^{\prime}, \xi^{\prime}, y, \eta, t\right) m^{(2)}\left(x^{\prime}, \xi^{\prime}, t\right) d x d \xi d x^{\prime} d \xi^{\prime} .
\end{array}\right.
$$

We focus next on $I_{1}(t)$ since $I_{2}(t)$ is handled similarly.

The classical proof of uniqueness for smooth paths uses $\rho^{s}(x-y) \rho^{s}\left(x^{\prime}-y\right) \rho^{v}(\xi-$ $\eta) \rho^{v}\left(\xi^{\prime}-\eta\right)$ in place of $\rho(x, \xi, y, \eta, t) \rho_{\varepsilon}\left(x^{\prime}, \xi^{\prime}, y, \eta, t\right)$. As a result, instead of $\partial_{\xi} \rho(x, \xi, y, \eta, t) \rho_{\varepsilon}\left(x^{\prime}, \xi^{\prime}, y, \eta, t\right)$, the integrand has $\partial_{\xi} \rho_{\varepsilon}^{v}(\xi-\eta) \rho_{\varepsilon}^{v}\left(\xi^{\prime}-\eta\right)$, and, hence, it is easy to interchange $\xi$ and $\xi^{\prime}$ derivatives to obtain cancellations. This is, 
however, not the case here and we need to introduce the appropriate derivatives, a fact that gives rise to additional terms and error terms that need to be approximated.

After integrating with respect to $y$ and $\eta$, we find

$$
I_{1}(t)=I_{11}(t)+I_{12}(t)
$$

with

$$
\begin{aligned}
I_{11}(t):= & -\int m^{(1)}(x, \xi, t) \chi^{(2)}\left(x^{\prime}, \xi^{\prime}, t\right) \rho_{\varepsilon}(x, \xi, y, \eta, t) \partial_{\xi^{\prime}} \\
& \times \rho_{\varepsilon}\left(x^{\prime}, \xi^{\prime}, y, \eta, t\right) d y d \eta d x d \xi d x^{\prime} d \xi^{\prime},
\end{aligned}
$$

and

$$
\begin{aligned}
I_{12}(t):= & \int m^{(1)}(x, \xi, y, \eta, t) \chi^{(2)}\left(x^{\prime}, \xi^{\prime}, y, \eta, t\right)\left[\partial_{\xi} \rho_{\varepsilon}(x, \xi, y, \eta, t) \rho_{\varepsilon}\left(x^{\prime}, \xi^{\prime}, y, \eta, t\right)\right. \\
& \left.+\rho_{\varepsilon}(x, \xi, y, \eta, t) \partial_{\xi^{\prime}} \rho_{\varepsilon}\left(x^{\prime}, \xi^{\prime}, y, \eta\right)\right] d y d \eta d x d \xi d x^{\prime} d \xi^{\prime} .
\end{aligned}
$$

Integrating by parts in $I_{11}(t)$ and using that $\partial_{\xi^{\prime}} \chi^{(2)}\left(x^{\prime}, \xi^{\prime}, t\right)=\delta\left(\xi^{\prime}\right)-$ $\delta_{u^{2}\left(x^{\prime}, t\right)}\left(\xi^{\prime}\right) \leq \delta\left(\xi^{\prime}\right)$, which is an immediate consequence of the definition of $\chi^{(2)}$ and the positivity, in the sense of distributions, of the Dirac mass, we find

$$
\begin{aligned}
I_{11}(t)= & \int m^{(1)}(x, \xi, t) \partial_{\xi^{\prime}} \chi^{(2)}\left(x^{\prime}, \xi^{\prime}, t\right) \rho_{\varepsilon}(x, \xi, y, \eta, t) \\
& \times \rho_{\varepsilon}\left(x^{\prime}, \xi^{\prime}, y, \eta, t\right) d y d \eta d x d \xi d x^{\prime} d \xi^{\prime} \leq I_{13}(t)
\end{aligned}
$$

with

$I_{13}(t):=\int m^{(1)}(x, \xi, t) \rho_{\varepsilon}(x, \xi, y, \eta, t)\left[\int \rho_{\varepsilon}\left(x^{\prime}, \xi^{\prime}, y, \eta, t\right) \delta\left(\xi^{\prime}\right) d \xi^{\prime} d x^{\prime}\right] d y d \eta d x d \xi$

Next we use that, in light of $\delta\left(\xi^{\prime}\right)=\frac{1}{2} \partial_{\xi^{\prime}} \operatorname{sgn}\left(\xi^{\prime}\right)$,

$$
\int \rho_{\varepsilon}\left(x^{\prime}, \xi^{\prime}, y, \eta, t\right) \delta\left(\xi^{\prime}\right) d \xi^{\prime} d x^{\prime}=-\frac{1}{2} \int \partial_{\xi^{\prime}} \rho_{\varepsilon}\left(x^{\prime}, \xi^{\prime}, y, \eta, t\right) \operatorname{sgn}\left(\xi^{\prime}\right) d \xi^{\prime} d x,
$$

and rewrite $I_{13}(t)$ as

$$
I_{13}(t)=I_{14}(t)+I_{15}(t)
$$

with

$$
\begin{aligned}
I_{14}(t):= & \frac{1}{2} \int m^{(1)}(x, \xi, t) \partial_{\xi} \rho_{\varepsilon}(x, \xi, y, \eta, t) \\
& \times\left[\int \rho_{\varepsilon}\left(x^{\prime}, \xi^{\prime}, y, \eta, t\right) \operatorname{sgn}\left(\xi^{\prime}\right) d \xi^{\prime} d x^{\prime}\right] d y d \eta d x d \xi
\end{aligned}
$$


and

$$
\begin{aligned}
I_{15}(t):= & -\frac{1}{2} \int m^{(1)}(x, \xi, t) \operatorname{sgn}\left(\xi^{\prime}\right)\left[\rho_{\varepsilon}\left(x^{\prime}, \xi^{\prime}, y, \eta, t\right) \partial_{\xi} \rho_{\varepsilon}(x, \xi, y, \eta, t)\right. \\
& \left.+\rho_{\varepsilon}(x, \xi, y, \eta, t) \partial_{\xi^{\prime}} \rho_{\varepsilon}\left(x^{\prime}, \xi^{\prime}, y, \eta, t\right)\right] d x^{\prime} d \xi^{\prime} d y d \eta d x d \xi .
\end{aligned}
$$

Since $\rho_{\varepsilon}(x, \xi, y, \eta, t)=\rho_{\varepsilon}^{s}\left(X_{(x, \xi)}-y\right) \rho_{\varepsilon}^{v}\left(\Xi_{(x, \xi)}-\eta\right)$, we find

$$
\begin{aligned}
\partial_{\xi} \rho_{\varepsilon}(x, \xi, y, \eta, t)= & -D_{y} \rho_{\varepsilon}^{s}\left(X_{(x, \xi)}-y\right) \rho_{\varepsilon}^{v}\left(\Xi_{(x, \xi)}-\eta\right) \partial_{\xi} X_{(x, \xi)} \\
& -\rho_{\varepsilon}^{s}\left(X_{(x, \xi)}-y\right) D_{\eta} \rho_{\varepsilon}^{v}\left(\Xi_{(x, \xi)}-\eta\right) \partial_{\xi} \Xi_{(x, \xi)} .
\end{aligned}
$$

We continue with two key observations. In light of (29) and the facts that

$$
\begin{aligned}
& \rho_{\varepsilon}\left(x^{\prime}, \xi^{\prime}, y, \eta, t\right)=\rho_{\varepsilon}^{s}\left(X_{\left(x^{\prime}, \xi^{\prime}\right)}-y\right) \rho_{\varepsilon}^{v}\left(\Xi_{\left(x^{\prime}, \xi^{\prime}\right)}-\eta\right), \\
& \int \rho_{\varepsilon}^{s}\left(x^{\prime}-y\right) d x^{\prime}=1 \text { and } d X_{\left(x^{\prime}, \xi^{\prime}\right)} d \Xi_{\left(x^{\prime}, \xi^{\prime}\right)}=d x^{\prime} d \xi^{\prime},
\end{aligned}
$$

the last being a consequence of the Hamiltonian structure of the system of characteristics, the first observation is that

$$
\begin{aligned}
\int \rho_{\varepsilon}\left(x^{\prime}, \xi^{\prime}, y, \eta, t\right) \operatorname{sgn}\left(\xi^{\prime}\right) d \xi^{\prime} d x^{\prime} & =\int \rho_{\varepsilon}\left(x^{\prime}, \xi^{\prime}, y, \eta, t\right) \operatorname{sgn}\left(\Xi_{\left(x^{\prime}, \xi^{\prime}\right)}\right) d \xi^{\prime} d x^{\prime} \\
& =\int \rho_{\varepsilon}\left(x^{\prime}, \xi^{\prime}, y, \eta, 0\right) \operatorname{sgn} \xi^{\prime} d \xi^{\prime} d x^{\prime} \\
& =\int \operatorname{sgn} \xi^{\prime} \rho_{\varepsilon}^{v}\left(\xi^{\prime}-\eta\right) d \xi^{\prime}
\end{aligned}
$$

The second is

$$
D_{\eta} \int_{\mathbb{R}} \operatorname{sgn}\left(\xi^{\prime}\right) \rho_{\varepsilon}^{v}\left(\xi^{\prime}-\eta\right) d \xi^{\prime}=2 \rho_{\varepsilon}^{v}(-\eta) \text { and } \int_{\mathbb{R}^{N}} D_{y} \rho_{\varepsilon}^{s}\left(X_{x, \xi}-y\right) d y=0 .
$$

Using the above in the expression for $I_{14}(t)$, after integrating by parts, we find

$$
I_{14}(t)=-\int m^{(1)}(x, \xi, t) \rho_{\varepsilon}^{v}(-\eta) \rho_{\varepsilon}(x, \xi, y, \eta, t) \partial_{\xi} \Xi_{(x, \xi)}
$$

Repeating this calculation for $I_{2}(t)$ adding the two inequalities, we get

$$
\begin{aligned}
& -\frac{d}{d t}\left[\rho_{\varepsilon} \star \chi^{(1)}(y, \eta, t) \rho_{\varepsilon} \star \chi^{(2)}(y, \eta, t)\right] \\
& \leq \int\left[m^{(1)}(x, \xi, t)+m^{(2)}(x, \xi, t)\right] \rho_{\varepsilon}^{v}(-\eta) \rho_{\varepsilon}(x, \xi, y, \eta, t) \partial_{\xi} \Xi_{(x, \xi)} d y d \eta d x d \xi \\
& \quad+\operatorname{Err}_{\varepsilon}^{(1)}(t)+\operatorname{Err}_{\varepsilon}^{(2)}(t),
\end{aligned}
$$


where

$$
\begin{aligned}
\operatorname{Err}_{\varepsilon}^{(1)}(t)= & \int\left[m^{(1)}(x, \xi, t) \chi^{(2)}\left(x^{\prime}, \xi^{\prime}, t\right)+m^{(2)}\left(x^{\prime}, \xi^{\prime}, t\right) \chi^{(1)}(x, \xi, t)\right] \\
& \times\left[\partial_{\xi} \rho_{\varepsilon}(x, \xi, y, \eta, t) \rho_{\varepsilon}\left(x^{\prime}, \xi^{\prime}, y, \eta, t\right)+\rho_{\varepsilon}(x, \xi, y, \eta, t) \partial_{\xi^{\prime}}\right. \\
& \left.\times \rho_{\varepsilon}\left(x^{\prime}, \xi^{\prime}, y, \eta\right)\right] d y d \eta d x d \xi d x^{\prime} d \xi^{\prime}
\end{aligned}
$$

and

$$
\begin{aligned}
\operatorname{Err}_{\varepsilon}^{(2)}(t):= & \int\left[m^{(1)}(x, \xi, t) \operatorname{sgn}\left(\xi^{\prime}\right)+m^{(2)}\left(x^{\prime}, \xi^{\prime}, t\right) \operatorname{sgn}(\xi)\right] \\
& \times\left[\partial_{\xi} \rho_{\varepsilon}(x, \xi, y, \eta, t) \rho_{\varepsilon}\left(x^{\prime}, \xi^{\prime}, y, \eta, t\right)+\rho_{\varepsilon}(x, \xi, y, \eta, t) \partial_{\xi^{\prime}}\right. \\
& \left.\times \rho_{\varepsilon}\left(x^{\prime}, \xi^{\prime}, y, \eta\right)\right] d y d \eta d x d \xi d x^{\prime} d \xi^{\prime}
\end{aligned}
$$

The other terms in $\frac{d}{d t} F_{\varepsilon}$. Since, for each $y$ and $\eta, q_{\varepsilon}(x, \xi, y, \eta, t)$ solves (18), for $i=1,2$, we have

$$
\frac{d}{d t} q_{\varepsilon} \star \chi^{(i)}(y, \eta, t)=-\int \partial_{\xi} q_{\varepsilon}(x, \xi, y, \eta, t) m^{(i)}(x, \xi, t) d x d \xi,
$$

and, in view of (32),

$$
\frac{d}{d t} q_{\varepsilon} \star \chi^{(i)}(y, \eta, t)=\int m^{(i)}(x, \xi, t) \rho_{\varepsilon}^{v}(-\eta) \rho_{\varepsilon}\left(x, \xi, y, \eta, t ; t_{0}\right) \partial_{\xi} \Xi_{(x, \xi)} d x d \xi
$$

The upper bound $\frac{d}{d t} F_{\varepsilon}$. Combining the previous two steps we conclude that, for almost every $t \in[0, h]$,

$$
\frac{d}{d t} F_{\varepsilon}(t) \leq \operatorname{Err}_{\varepsilon}^{(1)}(t)+\operatorname{Err}_{\varepsilon}^{(2)}(t)
$$

The estimate of the error terms. It is immediate that, for $0 \leq \tau \leq h$ and $i=1,2$,

$$
\int_{0}^{\tau}\left|\operatorname{Err}_{\varepsilon}^{(\mathrm{i})}(t)\right| d t \leq A(h, \varepsilon) \int_{0}^{\tau} \int\left[m^{(1)}(x, \xi, t)+m^{(2)}(x, \xi, t)\right] d x d \xi d t
$$

where

$$
A(h, \varepsilon):=\sup _{0 \leq t \leq h, x \in \mathbb{R}^{N}, \xi \in \mathbb{R}} B_{\varepsilon}(x, \xi, t)
$$

and

$$
\begin{aligned}
B_{\varepsilon}:= & \int \mid \int\left[\partial_{\xi} \rho_{\varepsilon}(x, \xi, y, \eta, t) \rho_{\varepsilon}\left(x^{\prime}, \xi^{\prime}, y, \eta, t\right)\right. \\
& \left.+\rho_{\varepsilon}(x, \xi, y, \eta, t) \partial_{\xi}^{\prime} \rho_{\varepsilon}\left(x^{\prime}, \xi^{\prime}, y, \eta\right)\right] d y d \eta \mid d x^{\prime} d \xi^{\prime}
\end{aligned}
$$


We show that there exists a uniform $C>0$ such that

$$
A(h, \varepsilon) \leq C \omega(h) .
$$

The estimate of $B_{\varepsilon}$. To prove (43) for $A_{\varepsilon}$ we observe that, in light of the properties of $\rho_{\varepsilon}^{s}$ and $\rho_{\varepsilon}^{v}$,

$$
\begin{aligned}
\int & {\left[\partial_{\xi} \rho_{\varepsilon}(x, y, \xi, \eta, t) \rho_{\varepsilon}\left(x^{\prime}, \xi^{\prime}, y, \eta\right)+\rho_{\varepsilon}(x, y, \xi, \eta, t) \partial_{\xi}^{\prime} \rho_{\varepsilon}\left(x^{\prime}, \xi^{\prime}, y, \eta\right)\right] d y d \eta } \\
= & \int\left[\partial_{\xi} X \cdot D \rho_{\varepsilon}^{s}(X-y) \rho_{\varepsilon}^{v}(\Xi-\eta) \rho_{\varepsilon}^{s}\left(X^{\prime}-y\right) \rho_{\varepsilon}^{s}\left(\Xi^{\prime}-\eta\right)\right. \\
& \left.+\rho_{\varepsilon}^{s}(X-y) \rho_{\varepsilon}^{s}(\Xi-\eta) \partial_{\xi} X^{\prime} \cdot D \rho_{\varepsilon}^{s}\left(X^{\prime}-y\right) \rho_{\varepsilon}^{v}\left(\Xi^{\prime}-\eta\right)\right] d y d \eta \\
& +\int\left[\rho_{\varepsilon}^{s}(X-y) \partial_{\xi} \Xi \cdot D \rho_{\varepsilon}^{v}(\Xi-\eta) \rho_{\varepsilon}^{s}\left(X^{\prime}-y\right) \rho_{\varepsilon}^{s}\left(\Xi^{\prime}-\eta\right)\right. \\
& \left.+\rho_{\varepsilon}^{s}(X-y) \rho_{\varepsilon}^{s}(\Xi-\eta) \rho_{\varepsilon}^{s}\left(X^{\prime}-y\right) \partial_{\xi} \Xi^{\prime} \cdot D \rho_{\varepsilon}^{v}\left(\Xi^{\prime}-\eta\right)\right] d y d \eta,
\end{aligned}
$$

where to simplify the notation we omitted for $X, \Xi, X^{\prime}$ and $\Xi^{\prime}$.

The integrands in the two terms behave similarly, hence, here, we study the first one. To this end, we first notice that $\partial_{\xi} X$ being independent of $y$ and $\eta$ can be factored out of the integral, while

$$
\begin{aligned}
\int_{\mathbb{R}^{N}} & {\left[D \rho_{\varepsilon}^{s}(X-y) \rho_{\varepsilon}^{v}(\Xi-\eta) \rho_{\varepsilon}^{s}\left(X^{\prime}-y\right) \rho_{\varepsilon}^{s}\left(\Xi^{\prime}-\eta\right)\right.} \\
& \left.+\rho_{\varepsilon}^{s}(X-y) \rho_{\varepsilon}^{s}(\Xi-\eta) D \rho_{\varepsilon}^{s}\left(X^{\prime}-y\right) \rho_{\varepsilon}^{v}\left(\Xi^{\prime}-\eta\right)\right] d y \\
= & -\int_{\mathbb{R}^{N}} D_{y}\left[\rho_{\varepsilon}^{s}(X-y) \rho_{\varepsilon}^{s}\left(X^{\prime}-y\right)\right] \rho_{\varepsilon}^{v}(\Xi-\eta) \rho_{\varepsilon}^{s}\left(\Xi^{\prime}-\eta\right) d y=0 .
\end{aligned}
$$

Therefore the term we need to estimate is

$$
\left|\left[\partial_{\xi} X-\partial_{\xi} X^{\prime}\right] \times \int D \rho_{\varepsilon}^{s}(X-y) \rho_{\varepsilon}^{v}(\Xi-\eta) \rho_{\varepsilon}^{s}\left(X^{\prime}-y\right) \rho_{\varepsilon}^{s}\left(\Xi^{\prime}-\eta\right) d y d \eta\right|,
$$

which is controlled by

$$
\left|\partial_{\xi} X-\partial_{\xi} X^{\prime}\right| \int\left|D \rho_{\varepsilon}^{s}(X-y)\right| \rho_{\varepsilon}^{v}(\Xi-\eta) \rho_{\varepsilon}^{s}\left(X^{\prime}-y\right) \rho_{\varepsilon}^{s}\left(\Xi^{\prime}-\eta\right) d y d \eta .
$$

It follows that $A(h, \varepsilon) \leq \sup _{\mathcal{A}}\left|\partial_{\xi} X-\partial_{\xi} X^{\prime}\right| \int\left|D \rho_{\varepsilon}^{s}(X-y)\right| \rho_{\varepsilon}^{v}(\Xi-\eta) \rho_{\varepsilon}^{s}\left(X^{\prime}-y\right) \rho_{\varepsilon}^{s}\left(\Xi^{\prime}-\eta\right) d y d \eta d x^{\prime} d \xi^{\prime}$, where $\mathcal{A}=:\left\{(x, \xi, t) \in \mathbb{R}^{N} \times \mathbb{R} \times(0, h]:\left|X(0)-X^{\prime}(0)\right| \leq C \varepsilon,\left|\Xi(0)-\Xi^{\prime}(0)\right| \leq C \varepsilon\right\}$. 
Since, in light of the Hamiltonian structure of the system of the characteristics, we have $d x^{\prime} d \xi^{\prime}=d X^{\prime} d \Xi^{\prime}$, there exists $C>0$ such that

$$
\begin{aligned}
& \int\left|D \rho_{\varepsilon}^{s}(X-y)\right| \rho_{\varepsilon}^{v}(\Xi-\eta) \rho_{\varepsilon}^{s}\left(X^{\prime}-y\right) \rho_{\varepsilon}^{s}\left(\Xi^{\prime}-\eta\right) d y d \eta d x^{\prime} d \xi^{\prime} \\
& =\int\left|D \rho_{\varepsilon}^{s}(X-y)\right| \rho_{\varepsilon}^{v}(\Xi-\eta) d y d \eta \leq C / \varepsilon
\end{aligned}
$$

and the estimate is reduced to showing that

$$
\sup _{\mathcal{A}}\left|\partial_{\xi} X-\partial_{\xi} X^{\prime}\right| C / \varepsilon \leq \omega(h)
$$

The last inequality follows from a cancelation property proved in [19], which yields that the characteristics (28) satisfy, with variable $s=W(t)$,

$$
\left|\partial_{\xi} X(s)-\partial_{\xi} X^{\prime}(s)\right| \leq C s \varepsilon,
$$

whenever the data at $s=0$ are $\varepsilon$ close.

\section{The existence of stochastic entropy solutions}

\subsection{The general strategy of the existence}

We consider a family of approximate problems using smooth local uniform approximations $W_{\varepsilon}$ of $W$, that is paths $W_{\varepsilon} \in C^{1}(\mathbb{R})$ such that, as $\varepsilon \rightarrow 0$ and for every $T>0$, $W_{\varepsilon} \rightarrow W$ uniformly on $[0, T]$. We construct pathwise stochastic entropy solutions as the limit of solutions to (3) with smooth paths.

Given $u^{0} \in\left(L^{1} \cap L^{\infty}\right)\left(\mathbb{R}^{N}\right)$ the conservation law (3) with $W_{\varepsilon}$ in place of $W$ has a unique entropy solution $u_{\varepsilon}$ with kinetic formulation

$$
\left\{\begin{array}{l}
\partial_{t} \chi_{\varepsilon}+\operatorname{div}_{x}\left[\mathbf{a}(x, \xi) \chi_{\varepsilon}\right] \dot{W}_{\varepsilon}-\frac{\partial}{\partial \xi}\left[b(x, \xi) \chi_{\varepsilon}\right] \dot{W}_{\varepsilon}=\partial_{\xi} m_{\varepsilon} \quad \text { in } \mathbb{R}^{N} \times \mathbb{R} \times(0, \infty), \\
\chi_{\varepsilon}=\chi\left(u^{0}(\cdot), \cdot\right) \text { on } \quad \mathbb{R}^{N} \times \mathbb{R} \times\{0\},
\end{array}\right.
$$

where, with the notation (12), $\chi_{\varepsilon}(x, \xi, t)=\chi\left(u_{\varepsilon}(x, t), \xi\right)$ and a measure $m_{\varepsilon}$ satisfying (14) and (16) with $\left\|u_{\varepsilon}(\cdot, t)\right\|_{\infty}$ in place of $\|u(\cdot, t)\|_{\infty}$ and, in principle, $C_{T}$ depending on $\varepsilon$.

To prove that the $u_{\varepsilon}$ 's converge, as $\varepsilon \rightarrow 0$, to a pathwise stochastic entropy solution of the sscl we need to obtain uniform in $\varepsilon$ a priori estimates, that is to show that, for each $T>0$, there exist $C_{T}, K_{T}>0$, which are independent of $\varepsilon$, such that, for all $t \in[0, T]$,

$$
\int m_{\varepsilon}(x, \xi, t) d x d \xi d t \leq C_{T}\left\|u^{0}\right\|_{2}^{2} \text { and }\left\|u_{\varepsilon}(\cdot, t)\right\|_{\infty}+\left\|u_{\varepsilon}(\cdot, t)\right\|_{1} \leq K_{T}
$$


As mentioned in Sect. 2, the $L^{1}$-bound always holds true, since in light of (6) and the positivity of $m_{\varepsilon}$, the contraction property for the $\varepsilon$ problem yields that

$$
\begin{aligned}
\frac{d}{d t} \int\left|u_{\varepsilon}(x, t)\right| d x & =\frac{d}{d t} \int\left|\chi_{\varepsilon}(x, t)\right| d x d \xi \\
& =-\dot{W}_{\varepsilon}(t) \int b(x, 0)\left|\chi_{\varepsilon}(x, t)\right| d x d \xi-\int m_{\varepsilon}(x, 0, t) d x \leq 0 .
\end{aligned}
$$

The $L^{\infty}$-estimate is more difficult to establish. At the end of this section we prove a general bound that only uses (7) as well as we discuss some examples not covered by it. We also present the bound on the total mass of $m_{\varepsilon}$; we remark that this is a new bound even for the theory of deterministic inhomogeneous scalar conservation laws.

\subsection{The limiting process}

In what follows we fix $T>0$ and work on $[0, T]$. We follow the construction based on weak limits proposed in Perthame [27,28], where we refer for many of the technical details, which relies on the kinetic formulation and is an alternative to the construction by Young measures in Di Perna [8].

Assuming (45) we can extract subsequences such that, as $\varepsilon \rightarrow 0$,

$$
u_{\varepsilon} \rightarrow u, \quad \chi_{\varepsilon} \rightarrow f \text { in } L^{\infty} \text { weak }-\star \text { and } m_{\varepsilon} \rightarrow m \text { in } M^{1} \text { weak }-\star
$$

with $u, f$ and $m$ also satisfying (16) and (45); here $M^{1}$ denotes the space of measures on $\mathbb{R}^{N} \times \mathbb{R} \times(0, \infty)$.

In addition some elementary structural properties of the nonlinear function $\chi$ in $\xi$ pass to the weak limit and give

$$
\operatorname{sgn} f(x, \xi, t)=\operatorname{sgn}(\xi),|f(x, \xi, t)| \leq 1 \text { and } \partial_{\xi} f(x, \xi, t)=\delta(\xi)-v(x, \xi, t)
$$

where $v(x, \xi, t) \geq 0$ is the Young measure associated with the weak- $\star$ limit of $u_{\varepsilon}$.

Passing to the weak limit in the definition of pathwise stochastic entropy solutions for (44) and using $\rho_{\varepsilon}(x, \xi, t)=\hat{\rho}\left(x, \xi, W_{\varepsilon}(t)-W_{\varepsilon}\left(t_{0}\right)\right)$ as test functions we obtain

$$
\left\{\begin{array}{l}
d f+\operatorname{div}_{x}[\mathbf{a}(x, \xi) f] \circ d W-\frac{\partial}{\partial \xi}[b(x, \xi) f] \circ d W=\partial_{\xi} m d t \text { in } \mathbb{R}^{N} \times \mathbb{R} \times(0, \infty), \\
f=\chi\left(u^{0}(\cdot), \cdot\right) \text { on } \quad \mathbb{R}^{N} \times \mathbb{R} \times\{0\} .
\end{array}\right.
$$

We leave it up to the reader to check that the definition of pathwise solutions applies to this equation following the lines of Sect. 2.

\subsection{The existence result}

The existence theorem is: 
Theorem 5.1 Assume (1), (2), (6), (7) and $u^{0} \in\left(L^{1} \cap L^{\infty}\right)\left(\mathbb{R}^{N}\right)$. There exists a pathwise stochastic entropy solution $u \in L^{\infty}\left((0, T) ;\left(L^{1} \cap L^{\infty}\right)\left(\mathbb{R}^{N}\right)\right)$, for all $T>0$, of (3) which is is continuous at $t=0$ and satisfies (14) and (16). The solution is given by $u(x, t)=\int_{\mathbb{R}} f(x, \xi, t) d \xi$ and $f(x, \xi, t)=\chi(u(x, t), \xi)$ solves (47) and satisfies (46).

Proof The existence of $u_{\varepsilon}$ has been already discussed and the $L^{\infty}$ - and $L^{2}$-bounds are proved below. Therefore, we may pass to the limit as indicated above and obtain the pathwise stochastic entropy solution $f$ of (47). It remains to prove that $f(x, \xi, t)=$ $\chi(u(x, t), \xi)$.

Consider the functional

$$
G(t):=\int\left[|f(x, \xi, t)|-f^{2}(x, \xi, t)\right] d x d \xi
$$

in place of $F(\cdot)$ in Sect. 4 and note that $G(0)=0$ because $f(x, \xi, 0)=\chi(x, \xi, 0)=$ $\chi\left(u^{0}(x), x\right)$. Following the same proof, we find that $G(t) \leq G(0)=0$ and, since $|f| \leq 1$, we conclude that $G \equiv 0$ and thus $f$ takes only the values 0 or 1 . In other words, in view of (46), $f$ is an indicator function like $\chi(u(x, t), \xi)$.

The continuity at time $t=0$ follows from a similar procedure (see [28], Proposition 4.1.7). For a sequence $t_{n} \rightarrow 0$, there is a weak limit $g(x, \xi)$ of $\chi\left(x, \xi, t_{n}\right)$, which satisfies (46) and, by the definition of pathwise stochastic solutions, $g \leq \chi\left(u^{0}(x), \xi\right)$. This means that $u\left(t_{n}\right)$ converges to $u^{0}$ strongly.

\subsection{The $L^{\infty}$-bound}

For $u^{0} \in L^{\infty}\left(\mathbb{R}^{N}\right)$ and $M=\left\|u^{0}\right\|_{\infty}$, we build, using the method of characteristics, a local in time smooth solution $U$ of

$$
\left\{\begin{array}{l}
U_{t}+\operatorname{div} \mathbf{A}(x, U)=0 \text { in } \mathbb{R}^{N} \times \mathbb{R} \\
U=M \text { on } \mathbb{R} \times\{0\} .
\end{array}\right.
$$

We show below that this smooth solution exists in $\mathbb{R}^{N} \times[-\tau, \tau]$ for some $\tau>0$ which does not depend on the size of $M$. We begin with the observation that mass conservation and the $L^{1}$ contraction imply the contraction property also on the positive and negative parts and, thus, a comparison principle. Therefore, it follows that $\left|u_{\varepsilon}(x, t)\right| \leq U\left(x, W_{\varepsilon}(t)\right)$ as long as $W_{\varepsilon}(t) \in[-\tau, \tau]$. In view of the uniform continuity of the paths, this last statement holds for $t \in\left[0, \tau^{*}\right]$ with $\tau^{*}$ depending only on $\tau$ and the modulus of continuity of the paths. Then we iterate the argument departing from the constant $\sup _{s \in[-\tau, \tau]}\|U(\cdot, s)\|_{\infty}$ and built the smooth large solution on $(\tau, 2 \tau),(2 \tau, 3 \tau)$, etc.. After a finite number of steps of order $\left(T / \tau^{*}+1\right)$, we reach the final time and obtain a uniform bound.

To construct the smooth solution on $[-\tau, \tau]$ we argue as follows. Departing from a constant $U(x, 0)=M$, the smooth solution of (48) is built by the method of characteristics (see (25)) with initial condition $Y(0)=x$ and $\zeta(0)=M$ as long as they do not intersect. This is possible as long as $\partial_{x} Y(t)$ is invertible. Notice that 
$\left(\partial_{x} Y, \partial_{x} \zeta\right)$ solves a system of differential equations (the linearization of (25) along $(Y, \zeta))$ with coefficients which, in view (7), are uniformly bounded independently of $M$. Since $\operatorname{det} \partial_{x} Y(0)=1$, the matrix $\partial_{x} Y(t)$ remains invertible for all $t \in[-\tau, \tau]$ for some uniform $\tau>0$. Because the solution is smooth, it generates a solution $U(x, W(t))$ for the stochastic equation (3) on the time interval $\left(0, \tau^{*}\right)$ with $\tau^{*}>0$ defined in the previous paragraph.

Next, we mention some special cases which do not fall in the general theory. A classical problem for applications is when there exist two ordered bounded steady states, that is smooth and bounded $k_{ \pm}$such that $k_{-} \leq k_{+}$and $\operatorname{div} A\left(x, k_{ \pm}(x)\right)=0$. If $k_{-} \leq u^{0} \leq k_{+}$, then we find the estimate

$$
\|u(t)\|_{\infty} \leq \max \left(\left\|k_{-}\right\|_{\infty},\left\|k_{+}\right\|_{\infty}\right) .
$$

For instance when $N=1$ and $A(x, u)=c(x) u^{2}$ with $c(x) \geq c_{m}>0$, one can choose $k_{ \pm}(x)= \pm \lambda_{ \pm} c(x)^{-1 / 2}$. When $N=2$ and $A(x, u)={ }^{\perp} \bar{D} V(x) B(u)$, one can choose constants. Another example, which is a model for multiphase flow in a porous medium, is $A(x, u)=V(x) u(1-u)$ and $k_{-}=0, k_{+}=1$ give the physical invariant region.

\subsection{The $L^{2}$-bound}

For $u^{0} \in L^{2}\left(\mathbb{R}^{N}\right)$, we prove the control of the total mass of the measure and employ again an iteration with uniform time steps.

We choose $\rho(x, \xi, 0)=\xi$. The solution $\rho(x, \xi, t)$ of the linear transport equation is $\rho(x, \xi, t)=\Xi_{(t, x, \xi)}(0)$, where as before $\left(X_{(t, x, \xi)}(0), \Xi_{(t, x, \xi)}(0)\right)$ are the backwards characteristics starting at $t$. From this representation, we conclude that there exists $\tau>0$ such that, for $0 \leq t \leq \tau, \partial_{\xi} \rho(x, \xi, t) \geq \frac{1}{2}$ and $\rho(x, \xi, t) \geq \frac{\xi}{2}$ for $\xi \geq 0$ and $\rho(x, \xi, t) \leq \frac{\xi}{2}$ for $\xi \leq 0$.

The definition of stochastic solution yields

$$
\begin{aligned}
& \int_{0}^{\tau} \int m_{\varepsilon}(x, \xi, t) \partial_{\xi} \rho(x, \xi, t) d x d \xi d t+\int \rho(x, \xi, \tau) \chi(x, \xi, t) d x d \xi \\
& \quad=\int \xi \chi(x, \xi, 0) d x d \xi=\frac{1}{2}\left\|u^{0}\right\|_{2}^{2} .
\end{aligned}
$$

The choice of $\tau$ and the properties of $\rho(x, \xi, t)$ imply immediately

$$
\frac{1}{2} \int_{0}^{\tau} \int m_{\varepsilon}(x, \xi, t) d x d \xi d t+\frac{1}{4}\|u(\cdot, \tau)\|_{2}^{2} \leq \frac{1}{2}\left\|u^{0}\right\|_{2}^{2}
$$

Given a final time $T$, we iterate, as in the proof of the $L^{\infty}$-bound, a finite number of times depending on the modulus of $W_{\varepsilon}(\cdot)$, which is, however, independent of $\varepsilon$ and we conclude. 
Acknowledgments We would like to thank Benjamin Gess for reading carefully a preliminary version of this note and pointing out several items that needed improvement. Panagiotis E. Souganidis was partially supported by the National Science Foundation Grants DMS-0901802 and DMS-1266383.

\section{References}

1. Berthelin, F., Vovelle, J.: Stochastic isentropic Euler equations (preprint, 2014)

2. Chen, G.-Q., Ding, Q., Karlsen, K.H.: On nonlinear stochastic balance laws. Arch. Ration. Mech. Anal. 204(3), 707-743 (2012)

3. Dafermos, C.M.: Hyperbolic Conservation Laws in Continuum Physics, Volume 325 of Grundlehren der Mathematischen Wissenschaften [Fundamental Principles of Mathematical Sciences], 3rd edn. Springer-Verlag, Berlin (2010)

4. Dalibard, A.-L.: Kinetic formulation for a parabolic conservation law. Application to homogenization. SIAM J. Math. Anal. 39(3), 891-915 (2007)

5. Debussche, A., Vovelle, J.: Invariant measure of scalar first-order conservation laws with stochastic forcing. arXiv:1310.3779v1 (preprint, 2013)

6. Debussche, A., Hofmanová, M., Vovelle, J.: Degenerate parabolic stochastic partial differential equations: Quasilinear case (preprint, 2013)

7. Debussche, A., Vovelle, J.: Scalar conservation laws with stochastic forcing. J. Funct. Anal. 259(4), 1014-1042 (2010)

8. Di Perna, R.J.: Measure-valued solutions to conservation laws. Arch. Ration Mech. Anal. 88(3), 223-270 (1985)

9. Feng, J., Nualart, D.: Stochastic scalar conservation laws. J. Funct. Anal. 255(2), 313-373 (2008)

10. Hofmanová, M.: Bhatnagar-Gross-Krook approximation to stochastic scalar conservation laws (preprint, 2013)

11. Hofmanová, M.: Degenerate parabolic stochastic partial differential equations. Stoch. Process. Appl. 123(12), 4294-4336 (2013)

12. Lasry, J.-M., Lions, P.-L.: Jeux à champ moyen. I. Le cas stationnaire. C. R. Math. Acad. Sci. Paris 343(9), 619-625 (2006)

13. Lasry, J.-M., Lions, P.-L.: Jeux à champ moyen. II. Horizon fini et contrôle optimal. C. R. Math. Acad. Sci. Paris 343(10), 679-684 (2006)

14. Lasry, J.-M., Lions, P.-L.: Mean field games. Jpn. J. Math. 2(1), 229-260 (2007)

15. Lions, P.-L., Perthame, B., Souganidis, P.E.: Existence and stability of entropy solutions for the hyperbolic systems of isentropic gas dynamics in Eulerian and Lagrangian coordinates. Commun. Pure Appl. Math. 49(6), 599-638 (1996)

16. Lions, P.-L., Perthame, B., Souganidis, P.E.: Weak stability of isentropic gas dynamics for $\gamma=5 / 3$. In: Progress in Elliptic and Parabolic Partial Differential Equations (Capri, 1994), Volume 350 of Pitman Res. Notes Math. Ser., pp. 184-192. Longman, Harlow (1996)

17. Lions, P.-L., Perthame, B., Souganidis, P.E.: Scalar conservation laws with rough (stochastic) fluxes (in preparation, 2013)

18. Lions, P.-L., Perthame, B., Tadmor, E.: A kinetic formulation of multidimensional scalar conservation laws and related equations. J. Am. Math. Soc. 7(1), 169-191 (1994)

19. Lions, P.-L., Souganidis, P.E.: Stochastic viscosity solutions (in preparation)

20. Lions, P.-L., Souganidis, P.E.: Fully nonlinear stochastic partial differential equations. C. R. Acad. Sci. Paris Ser. I Math. 326(9), 1085-1092 (1998)

21. Lions, P.-L., Souganidis, P.E.: Fully nonlinear stochastic partial differential equations: non-smooth equations and applications. C. R. Acad. Sci. Paris Ser. I Math. 327(8), 735-741 (1998)

22. Lions, P.-L., Souganidis, P.E.: Équations aux dérivées partielles stochastiques nonlinéaires et solutions de viscosité. In: Seminaire: Équations aux Dérivées Partielles, 1998-1999, Sémin. Équ. Dériv. Partielles, p. Exp. No. I, 15. École Polytech., Palaiseau (1999)

23. Lions, P.-L., Souganidis, P.E.: Fully nonlinear stochastic pde with semilinear stochastic dependence. C. R. Acad. Sci. Paris Ser. I Math. 331(8), 617-624 (2000)

24. Lions, P.-L., Souganidis, P.E.: Uniqueness of weak solutions of fully nonlinear stochastic partial differential equations. C. R. Acad. Sci. Paris Ser. I Math. 331(10), 783-790 (2000) 
25. Lions, P.-L., Souganidis, P.E.: Viscosity solutions of fully nonlinear stochastic partial differential equations. Sūrikaisekikenkyūsho Kōkyūroku 1287, 58-65 (2002). Viscosity solutions of differential equations and related topics (Japanese) (Kyoto, 2001)

26. Lyons, T.J.: Differential equations driven by rough signals. Rev. Mat. Iberoam. 14(2), 215-310 (1998)

27. Perthame, B.: Uniqueness and error estimates in first order quasilinear conservation laws via the kinetic entropy defect measure. J. Math. Pures Appl. 77(10), 1055-1064 (1998)

28. Perthame, B.: Kinetic Formulation of Conservation Laws, Volume 21 of Oxford Lecture Series in Mathematics and its Applications. Oxford University Press, Oxford (2002)

29. Perthame, B., Tadmor, E.: A kinetic equation with kinetic entropy functions for scalar conservation laws. Commun. Math. Phys. 136(3), 501-517 (1991)

30. Serre, D.: Systems of Conservation Laws, vol. 1. Cambridge University Press, Cambridge (1999). Hyperbolicity, entropies, shock waves, Translated from the 1996 French original by I. N. Sneddon 